\title{
Verbände, Veranstaltungen, Arbeitsvorhaben
}

\section{International Congress of Ecology}

Der Kongreß findet vom 10.-16. August 1986 an der State University of New York, College of Environmental Science and Forestry in Syracuse, New York 132210 USA, statt. Anmeldeformulare sind vom Veranstalter zu erhalten. Ein Zirkular informiert über Möglichkeiten, Vorträge oder Posterdemonstrationen anzumelden. Das Generalthema lautet: Global Connections in Ecological Theory and Practice.

\section{The Working Group on Granivorous Birds}

Während des IV. Internationalen Ökologenkongresses in Syracuse (s. oben) hält die Working Group on Granivorous Birds am 13. August 1986 ihr General Meeting als Symposium unter dem Thema "The role of granivorous birds in ecosystems". Das Programm wird im Dezember 1985 zusammengestellt. Information: Chairman of Working Group - Prof. Dr. Jan Pinowski, Department of Vertebrate Ecology, Institute of Ecology PAS, Dziekanów Leśny, 05-092 Łomianki, Poland.

\section{Dachverband Deutscher Avifaunisten}

Auf seiner Jahrestagung und Mitgliederversammlung am 2./3. März 1985 hat der DDA Herrn Dr. Brixo Ullerich den Preis zur Förderung der Feldornithologie 1985 zuerkannt für seine Arbeit „Zur Populationsdynamik des Steinkauzes" (Vogelwarte 30, 1980: 179-197). Der Preis besteht aus einer Verleihungsurkunde und DM 1000,- .

\section{East African Rare Birds Comittee}

Beobachtungen und Nachweise seltener Vogelarten in Ostafrika sollten vor Publikation der neu gebildeten Seltenheitskommission der East African Natural History Society vorgelegt werden, der 10 Experten angehören. Ein Seltenheitsreport erscheint jährlich als Teil 5 der Zeitschrift Scopus. Kontaktadresse: EANHS, c/o Dept. of Biochemistry, University of Nairobi, Box 30 197, Nairobi, Kenia. 